\title{
Autophagy positively regulates Wnt signaling in mice with diabetic retinopathy
}

\author{
SIHAO YE ${ }^{1 *}$, YUHAN ZHANG $^{1 *}$, XIN WANG $^{1}$, XU LIANG $^{1}$, MINGYAN WEI $^{1}$, \\ RONGRONG ZONG ${ }^{1}, \mathrm{ZUGUO} \mathrm{LIU}^{1-3}$ and QIAN CHEN ${ }^{1-3}$ \\ ${ }^{1}$ Provincial Key Laboratory of Ophthalmology and Visual Science, Eye Institute of Xiamen University, \\ School of Medicine, Xiamen University, Xiamen, Fujian 361102; ${ }^{2}$ Department of Ophthalmology, \\ Xiang'an Hospital of Xiamen University, Xiamen, Fujian 361101; ${ }^{3}$ Department of Ophthalmology, \\ Xiamen University Affiliated Xiamen Eye Center, Xiamen, Fujian 361100, P.R. China
}

Received March 30, 2021; Accepted July 16, 2021

DOI: $10.3892 /$ etm.2021.10598

\begin{abstract}
Diabetic retinopathy (DR) is a microvascular complication of diabetes. Aberrant Wnt signaling activation plays a pathological role in DR. However, the underlying mechanisms of aberrant Wnt signaling in DR remain unknown. Autophagy has been reported to be involved in the pathophysiology of DR. The present study aimed therefore to investigate the regulatory effects of autophagy on Wnt signaling in DR. Wnt signaling was activated in the retina of $\mathrm{db} / \mathrm{db}$ mice combined with an increase in the expression of the autophagic proteins microtubule-associated protein 1A/1B-light chain 3 and beclin-1 and a decrease in the expression of the autophagic protein P62. Inhibition of autophagy by 3-methyladenin decreased Wnt signaling in diabetic retinas, indicating a potential association between Wnt signaling and autophagy. Rapamycin, an autophagy inducer, upregulated Wnt signaling in the retina of normal C57BL/6J mice. In cultured Müller cells, rapamycin induced autophagy and activated Wnt signaling, while chloroquine, an autophagy inhibitor, inhibited autophagy and downregulated Wnt signaling, suggesting that autophagy could regulate Wnt signaling in mice retina and retinal cells. In summary, this study demonstrated that autophagy may positively regulate Wnt signaling in diabetic retinas, indicating a potential mechanism of Wnt signaling upregulation in DR and a possible novel therapeutic target of DR.
\end{abstract}

Correspondence to: Professor Zuguo Liu or Dr Qian Chen, Provincial Key Laboratory of Ophthalmology and Visual Science, Eye Institute of Xiamen University, School of Medicine, Xiamen University, 4221 South Xiang'an Road, Xiamen, Fujian 361102, P.R. China

E-mail: zuguoliu@xmu.edu.cn

E-mail: qchen2@xmu.edu.cn

${ }^{*}$ Contributed equally

Key words: autophagy, Wnt signaling, diabetic retinopathy, rat Müller cell, rapamycin, 3-methyladenin, chloroquine

\section{Introduction}

Diabetic retinopathy (DR) is a microvascular complication of diabetes and a major cause of vision loss in Western countries. The incidence of diabetes worldwide will reach $>366$ million by the end of 2030 (1). It is reported that nearly all patients with type 1 diabetes will develop some manifestation of DR, and that $\sim 50-80 \%$ of patients with type 2 diabetes will have DR within 20-25 years of developing the condition (2). With the growing population of individuals with diabetes, the incidence of DR will increase gradually, which poses a major threat to the global population and a costly burden to health care systems. The exact mechanism of DR is unclear, and the current treatments for diabetic retinopathy, such as anti-vascular endothelial growth factors and laser coagulation, have limited efficacies for patients with DR (3). Wnt signaling is an evolutionally conserved signaling pathway that serves essential roles in tissue development and adult homeostasis (4). Dysregulated Wnt signaling plays pathogenic roles in a variety of human diseases, including DR $(5,6)$. Upregulated Wnt signaling increases retinal inflammation and secretion of vascular endothelial growth factor (VEGF) and results in retinal neovascularization in diabetic animal models $(5,7)$. Blockage of the Wnt signaling pathway has anti-angiogenic and anti-inflammatory effects in DR $(8,9)$. However, the underlying mechanism of aberrant Wnt signaling activation in DR remains to be determined.

Autophagy is a catabolic process in which cellular components are degraded by lysosomes (10). Autophagy is essential for retinal development and vision formation (11). Previous studies have demonstrated that autophagy is involved in the pathogenesis of DR (12-14). For example, retinal autophagy is upregulated in diabetic human and mice, where it has been demonstrated to play dual roles in $\mathrm{DR}$, a protective role in mild stress [induced by $50 \mathrm{mg} / \mathrm{l}$ in vitro-modified heavily-oxidized glycated LDL (HOG-LDL)] in cultured human retinal capillary cells and a detrimental role in severe stress $(200 \mathrm{mg} / \mathrm{l}$ HOG-LDL) (13). High glucose increases endoplasmic reticulum stress and induces autophagy in Müller cells (14).

The interplay between autophagy and Wnt signaling has been previously reported. For example, Kallistatin, which is a 
Wnt signaling inhibitor, can induce apoptosis and autophagy in breast cancer cells, thereby reducing the rates of tumor angiogenesis and vascular growth (15). A previous study on lung cancer demonstrated that the tumor suppressor candidate 3 protein could induce expression of autophagy related proteins, which activates $\mathrm{Wnt} / \beta$-catenin signaling in human non-small lung cancer cells (16). The association between autophagy and Wnt signaling in cancer field is well established (17). However, whether autophagy might regulate Wnt signaling in DR has not yet been investigated.

The present study aimed to investigate the possible mechanism of abnormal Wnt signaling in DR and hypothesized that autophagy may have a regulatory effect on Wnt signaling in DR. Meanwhile, the investigation of autophagy in DR may shed a light on how autophagy was changed in DR and the possible function of autophagy in the retina.

\section{Materials and methods}

Animals. Heterozygous BKS.Cg-Dock $7 \mathrm{~m}^{+} /^{+}$Leprdb/J mice (stock no. 000642) were purchased from Jackson Laboratory. Homozygous $\mathrm{db} / \mathrm{db}$ mice and control mice were obtained by crossing heterozygous $\mathrm{db} /+$ mice. C57BL/6J [wild-type (WT)] mice were purchased from the Laboratory Animal Center of Xiamen University. All animals were housed in a specific-pathogen-free facility and maintained in 12-h light/dark cycle. All animal procedures were performed in accordance with the Association for Research in Vision and Ophthalmology 'Statement for the Use of Animals in Ophthalmic and Vision Research'. The animal protocols were approved by the Xiamen University Experimental Animal Ethics Committee (approval no. XMULA20190022).

Cell culture. The rat Müller (rMC-1) cell line was a gift from Dr Vijay Sarthy of Northwestern University (Evanston, IL, USA) and cultured in Dulbecco's modified Eagle's medium supplemented with $10 \%$ fetal calf serum and $1 \%$ penicillin streptomycin solution (all from Gibco; Thermo Fisher Scientific, Inc.) and placed at $37^{\circ} \mathrm{C}$ in a humidified incubator containing $5 \% \mathrm{CO}_{2}$.

Induction/inhibition of autophagy in vitro. rMC-1 cells were plated in a 6 -well plate at a density of $5 \times 10^{5} /$ well. After $24 \mathrm{~h}$, rMC-1 cells were treated with rapamycin $(4 \mu \mathrm{M})$ which was dissolved in DMSO (both from Sigma-Aldrich; Merck KGaA) or with the same amount of DMSO as a vehicle for $24 \mathrm{~h}$. To suppress the autophagy in rMC-1 cells, chloroquine $(1 \mu \mathrm{M}$; Sigma-Aldrich) was used to treat rMC-1 cells for $6 \mathrm{~h}$ and DMSO was used as vehicle control.

Induction/inhibition of autophagy in vivo. An autophagy inhibitor 3-methyladenin (3-MA; Sigma-Aldrich; Merck KGaA; $25 \mathrm{mg} / \mathrm{kg}$ ) was dissolved in DMSO and intraperitoneally injected into $\mathrm{db} / \mathrm{db}$ mice (5 months old; average weight, $54 \mathrm{~g}$ ) every other day for 30 days (18), while the same amount of DMSO was used as vehicle control in 5-month-old $\mathrm{db} / \mathrm{db}$ mice with the similar manner. Similarly, rapamycin (Sigma-Aldrich; Merck KGaA; $25 \mathrm{mg} / \mathrm{kg}$ ) was dissolved in DMSO and intraperitoneally injected into C57 mice ( 3 months old; average weight, $28 \mathrm{~g}$ ) once a day for 30 days to induce autophagy, while DMSO was used as vehicle control (19). At least six mice were used in each group. Mice were sacrificed using carbon dioxide (30\% cage volume $/ \mathrm{min}$ ) and the retinas were collected for subsequent experiments and stored in a $-80^{\circ} \mathrm{C}$ freezer for further use.

Western blotting. The treated rMC-1 cells and retinal tissues were lysed by radioimmunoprecipitation assay (RPAP) buffer (Sigma-Aldrich; Merck KGaA) and the total protein concentrations were measured by bicinchoninic acid assay. Each amount of protein ( $25 \mu \mathrm{g}$ per lane) was resolved by electrophoresis through $12 \%$ Tris-glycine SDS polyacrylamide gel and electrotransferred onto a polyvinylidene difluoride membrane. The membrane was blocked with $5 \%$ non-fat dry milk in Tris-buffered saline with $0.1 \%$ Tween (TBST)-20 for $2 \mathrm{~h}$ at room temperature. The membrane was incubated with the primary antibodies (dilution $1: 1,000$ ) at $4^{\circ} \mathrm{C}$ overnight. The primary antibodies against microtubule-associated protein 1A/1B-light chain 3B (LC3B; cat. no. ab48394), P62 (cat. no. ab56416) and VEGF (cat. no. ab46154) were obtained from Abcam. The primary antibodies against Beclin-1 (cat. no. sc-48341) and $\beta$-catenin (cat. no. sc-7199) were purchased from Santa Cruz Biotechnology, Inc. Primary antibodies against non-phosphorylated- $\beta$-catenin (n-p- $\beta$-catenin; cat. no. $4270 \mathrm{~S}$ ) and $\beta$-actin (cat. no. 4970) were purchased from Cell Signaling Technology, Inc. After three washes with TBST, the membranes were incubated with 1:5,000 dilution of a HRP-conjugated goat anti-mouse antibody (Sigma-Aldrich, cat. no. A4416) or an HRP-conjugated anti-rabbit antibody (Sigma-Aldrich; Merck KGaA; cat. no. A6154) in TBST containing 1\% dry milk for $1 \mathrm{~h}$. After four washes with TBST, the bands were detected using super ECL detection Reagent (Shanghai Yeasen Biotechnology Co., Ltd.) and the band intensities were semi-quantified by densitometry using Quantity One-1D analysis software (Bio-Rad Laboratories, Inc.).

Statistical analysis. GraphPad Prism version 7 (GraphPad Software, Inc.) was used for statistical analyses. Data were presented as the means \pm standard error of the mean. Comparison between two groups was performed using Student's t-test. At least three independent measurements were conducted for each assay. $\mathrm{P}<0.05$ was considered to indicate a statistically significant difference.

\section{Results}

Wnt signaling is activated and autophagy is stimulated in retinas from $\mathrm{db} / \mathrm{db}$ mice. Previous studies reported that Wnt signaling is abnormally activated in diabetic retinas and serves a pathogenic role in DR $(7,20)$. In the present study, $\mathrm{db} / \mathrm{db}$ mouse, a genetic type 2 diabetic animal model, was selected as animal model of diabetic retinopathy (21). The protein expression of non-p- $\beta$ catenin and $\beta$-catenin was evaluated by western blotting (Fig. 1A) and non-p- $\beta$ catenin expression was found to be significantly increased in $\mathrm{db} / \mathrm{db}$ retinas (Fig. 1B), while $\beta$-catenin expression was unchanged (Fig. 1C). Protein expression of the target gene of Wnt signaling VEGF was also significantly upregulated in $\mathrm{db} / \mathrm{db}$ retinas compared with those in control retinas (Fig. 1D and E). Furthermore, the expression of the autophagic proteins LC3BI, LC3BII (Fig. 1F), Beclin-1 (Fig. 1H) 
A

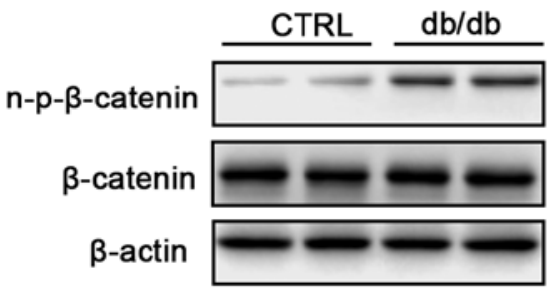

D

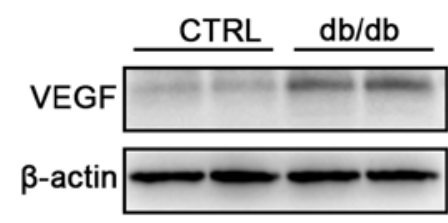

G

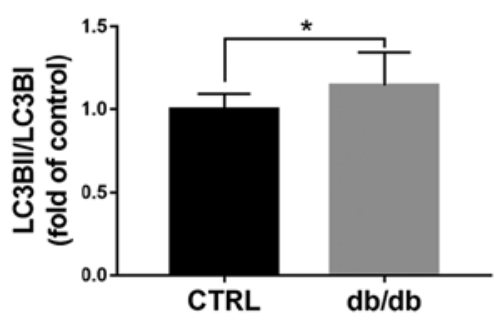

$J$

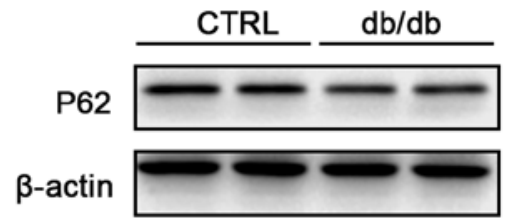

B

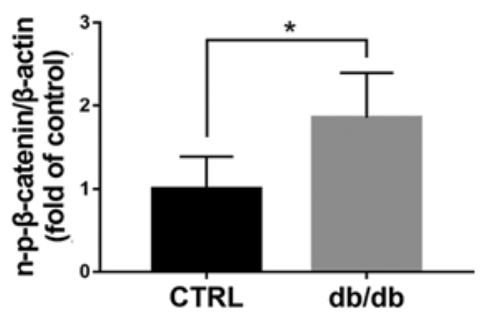

E

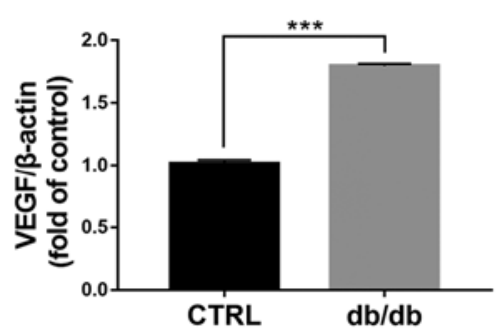

$\mathrm{H}$

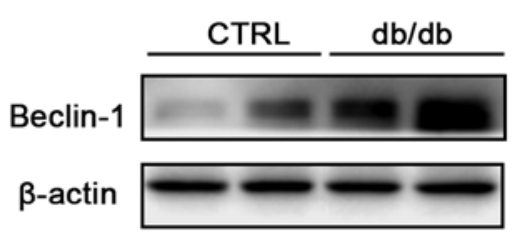

K

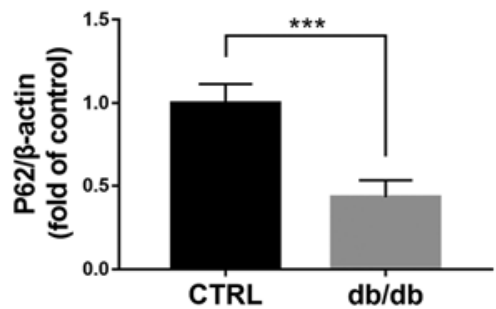

C

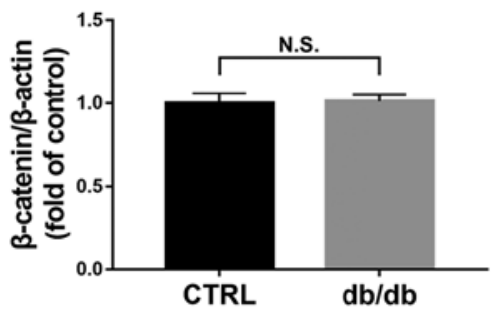

$\mathrm{F}$

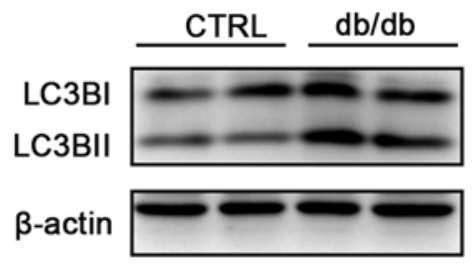

I

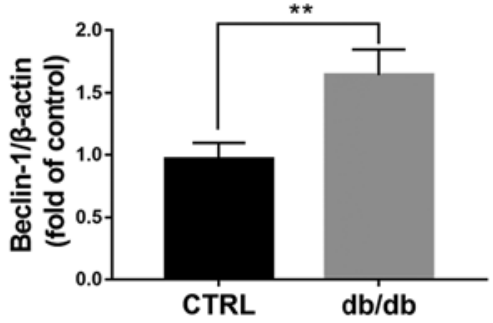

Figure 1. Positive relationship between Wnt signaling pathway and autophagy in $\mathrm{db} / \mathrm{db}$ retinas. (A) Expression of $n-p-\beta$-catenin and $\beta$-catenin was evaluated by western blotting in the retinas of WT and $\mathrm{db} / \mathrm{db}$ mice. (B) $n$-p- $\beta$-catenin and (C) $\beta$-catenin were quantified by densitometry and normalized to $\beta$-actin levels $(n=6)$. ( $D$ and E) Expression of the downstream target gene of Wnt signaling VEGF was evaluated and quantified in WT or db/db retinas ( $\mathrm{n}=6)$. Expression of (F) LC3BI and LC3BII (LC3BI/II), (H) Beclin-1 and (J) P62 was evaluated by western blotting. (G) Level of LC3BI/II was quantified by densitometry and the band intensity ratio of LC3BII to LC3BI (LC3BII/LC3BI) was calculated and compared (n=6). Levels of (I) Beclin-1 and (K) P62 were quantified by densitometry and normalized to $\beta$-actin levels $(\mathrm{n}=6) .{ }^{*} \mathrm{P}<0.05,{ }^{* *} \mathrm{P}<0.01$ and ${ }^{* * * *} \mathrm{P}<0.001$. n-p- $\beta$-catenin, non-phosphorylated- $\beta$-catenin; N.S., non-significant; $\mathrm{db}$, diabetic; LC3B, microtubule-associated protein 1A/1B-light chain 3; WT, wild-type; VEGF, vascular endothelial growth factor.

and P62 (Fig. 1J) was determined by western blotting. The ratio LC3BII/LC3BI (Fig. 1G) and expression of Beclin-1 (Fig.1I) were significantly elevated, whereas P62 expression (Fig. 1K) was significantly reduced in $\mathrm{db} / \mathrm{db}$ retinas compared with the control group, indicating an induction of autophagy in the retina of $\mathrm{db} / \mathrm{db}$ mice. Taken together, these findings indicated that Wnt signaling and autophagy in $\mathrm{db} / \mathrm{db}$ retinas may be related.

Autophagy inhibition downregulates Wnt signaling in $d b / d b$ retinas. To further clarify the relationship between autophagy and Wnt signaling, $\mathrm{db} / \mathrm{db}$ mice were treated with 3-MA, a selective PI3K inhibitor that inhibits autophagy (22). After one-month treatment, the ratio LC3BII/LC3BI (Fig. 2A and B) and Beclin-1 expression (Fig. 2C and D) were significantly decreased, whereas P62 expression was significantly increased in the retinas of $\mathrm{db} / \mathrm{db}$ mice treated with 3-MA compared with those treated with vehicle (Fig. 2C and E), suggesting a successful inhibition of autophagy in $\mathrm{db} / \mathrm{db}$ retinas. Furthermore, the protein expression of $n-p-\beta$-catenin was significantly decreased in the retinas of 3-MA-treated $\mathrm{db} / \mathrm{db}$ mice compared with the retinas of vehicle-treated $\mathrm{db} / \mathrm{db}$ mice (Fig. 2F and G), and the protein expression of $\beta$-catenin (Fig.2G and H) was unchanged. Protein expression of VEGF 
A

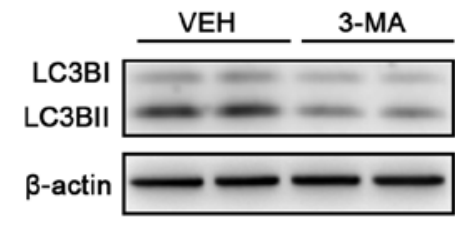

D

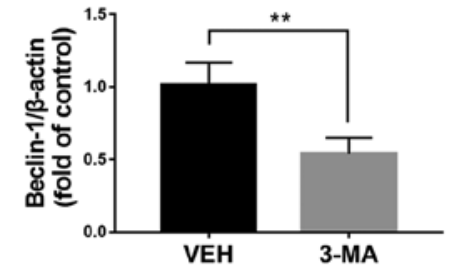

G

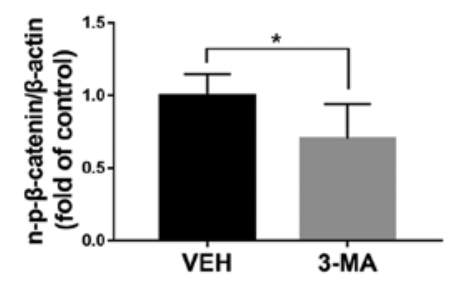

$\mathrm{J}$

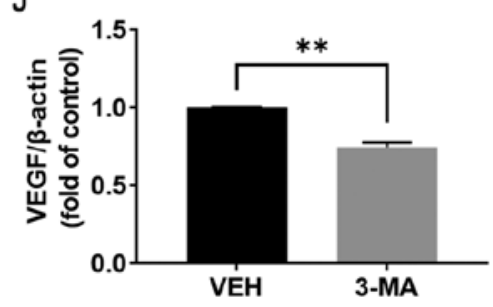

B

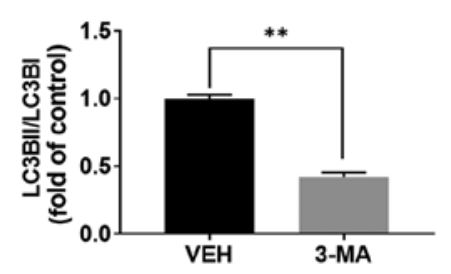

$E$

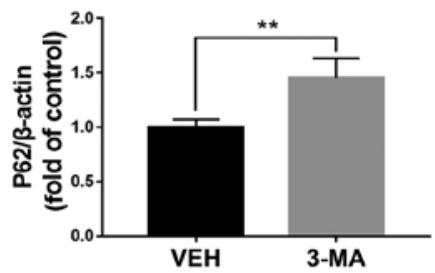

$\mathrm{H}$

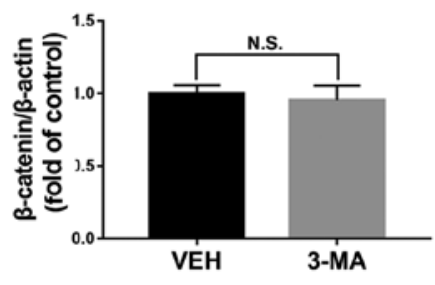

C

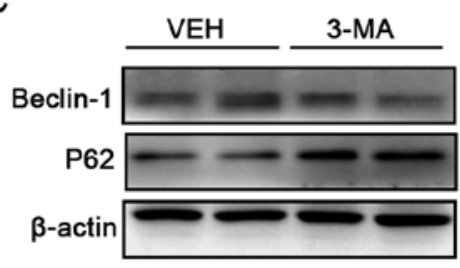

F

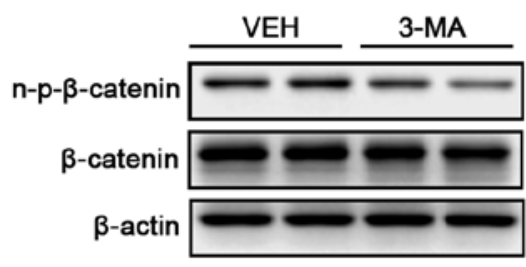

I

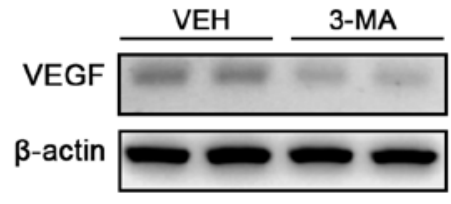

Figure 2. Inhibition of autophagy downregulates Wnt signaling in db/db retinas. Representative images of western blotting for (A) LC3BI/II, (C) Beclin-1, P62, (F) n-p- $\beta$-catenin and $\beta$-catenin in db/db mice treated with VEH or 3-MA. (B) Protein levels of LC3BI/II were quantified by densitometry and LC3BII/LC3BI was calculated and compared (n=6). Protein levels of (D) Beclin-1, (E) P62, (G) n-p- $\beta$-catenin and (H) $\beta$-catenin were quantified by densitometry and normalized to $\beta$-actin levels $(n=6)$. (I and J) Protein expression of the downstream target gene of Wnt signaling VEGF was evaluated and quantified in $\mathrm{db} / \mathrm{db}$ mice treated with VEH or 3-MA $(\mathrm{n}=6) .{ }^{*} \mathrm{P}<0.05$ and $^{* * *} \mathrm{P}<0.01$. n-p- $\beta$-catenin, non-phosphorylated- $\beta$-catenin; N.S., non-significant; LC3B, microtubule-associated protein 1A/1B-light chain 3; VEGF, vascular endothelial growth factor; VEH, vehicle; 3-MA, 3-methyladenin; db, diabetic.

was significantly decreased in the retinas of 3-MA-treated $\mathrm{db} / \mathrm{db}$ mice (Fig. 2I and J). In addition, the weight and blood glucose levels of $\mathrm{db} / \mathrm{db}$ mice were not significantly changed before or after treatment of 3-MA (Table SI). Taken together, these results demonstrated that inhibition of autophagy may be accompanied with inactivation of Wnt signaling, indicating a regulatory effect of autophagy on Wnt signaling.

Induction of autophagy activates Wnt signaling in WT retinas. To further investigate the effect of autophagy on the modulation of Wnt signaling, rapamycin was used to induce autophagy in normal WT retinas and the expression of proteins associated with Wnt signaling was evaluated by western blotting. Following administration of rapamycin, the ratio LC3BII/LC3BI (Fig. 3A and B) and expression of Beclin-1 (Fig.3C and D) were significantly increased, while P62 expression was significantly decreased in the retinas (Fig. 3E and F), suggesting the successful induction of autophagy in the retinas of WT mice. In addition, protein expression of n-p- $\beta$-catenin (Fig.3G and $\mathrm{H}$ ) was significantly increased in the retinas of rapamycin-treated WT retinas compared with those of vehicle-treated WT mice and the expression of $\beta$-catenin was unchanged (Fig. 3G and I). Furthermore, protein expression of VEGF was significantly increased in rapamycin-treated retinas (Fig. 3J and K). Taken together, these findings suggested that induction of autophagy may activate Wnt signaling in the retina.

Induction of autophagy upregulates Wnt signaling in rMC-1 cells. Müller cells are the primary glial cells that contribute to the pathological responses in DR (23). A previous study demonstrated that inactivation of Wnt signaling in Müller cells attenuates inflammatory responses in diabetic retinas (24). To determine on which cells autophagy may 
A

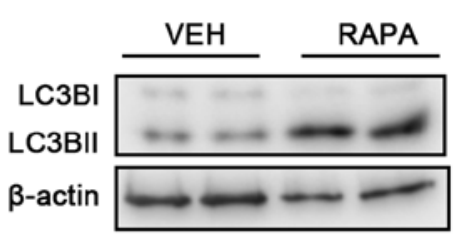

D

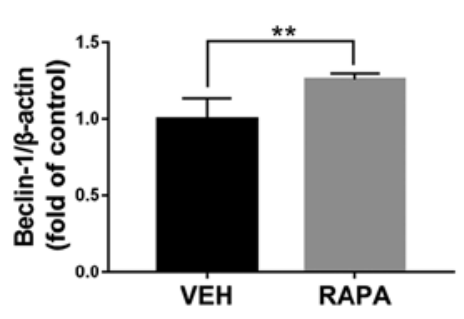

G

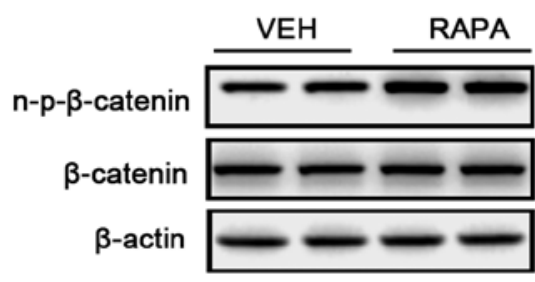

$J$

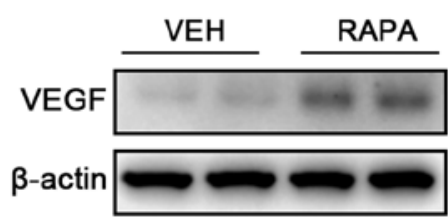

B

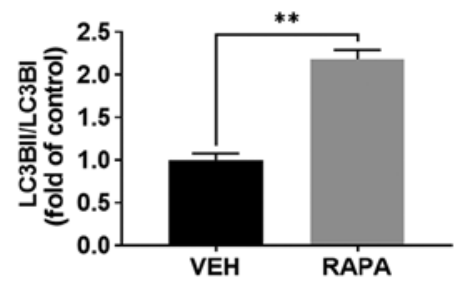

$E$

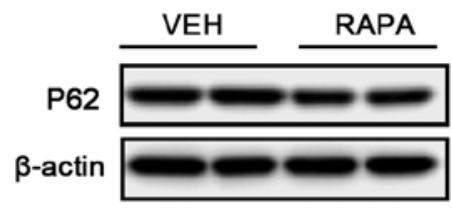

$\mathrm{H}$

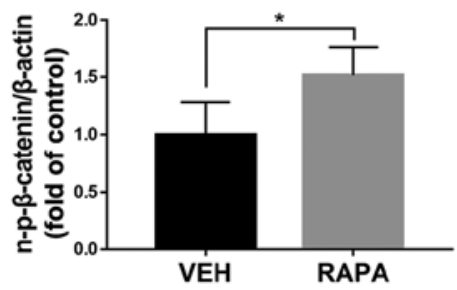

$\mathrm{K}$

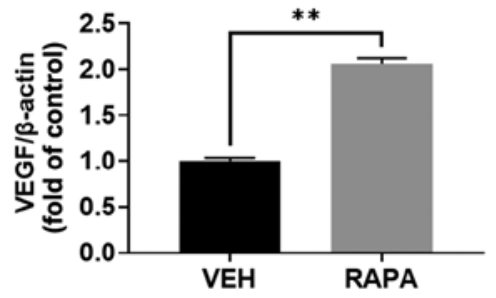

C

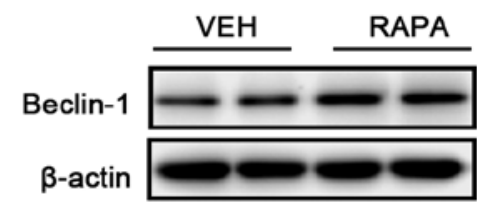

$\mathrm{F}$

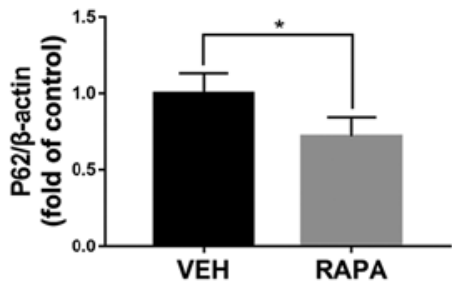

I

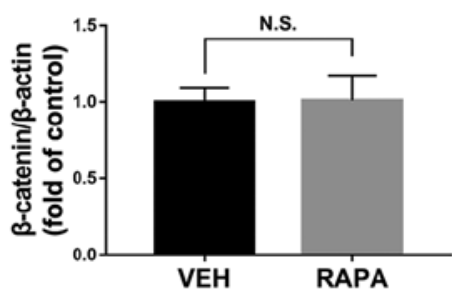

Figure 3. Induction of autophagy by RAPA activates Wnt signaling in C57BL/6J retinas. Expression of autophagy proteins (A) LC3BI/II, (C) Beclin-1 and (E) P62 was evaluated by western blotting in the retina of C57BL/6J mice treated with VEH or RAPA. (B) Protein levels of LC3BI/II were quantified by densitometry and LC3BII/LC3BI were calculated and compared ( $\mathrm{n}=6)$. Protein levels of (D) Beclin-1 and (F) P62 were quantified by densitometry and normalized to $\beta$-actin levels $(n=6)$. (G) Expression of $n-p-\beta$-catenin and $\beta$-catenin was evaluated by western blotting in the retina of C57BL/6J mice treated with VEH or RAPA. (H and I) Levels of $(\mathrm{H}) \mathrm{n}$-p- $\beta$-catenin and (I) $\beta$-catenin were quantified by densitometry and normalized to $\beta$-actin levels (n=6). (J and K) Expression of the downstream target gene of Wnt signaling VEGF was evaluated and quantified in the retina of C57BL/6J mice treated with VEH or RAPA ( $=6$ ). ${ }^{*} \mathrm{P}<0.05$ and ${ }^{* *} \mathrm{P}<0.01$. n-p- $\beta$-catenin, non-phosphorylated- $\beta$-catenin; N.S., non-significant; LC3B, microtubule-associated protein 1A/1B-light chain 3; VEGF, vascular endothelial growth factor; VEH, vehicle; RAPA, rapamycin.

have an effect, rMC-1 cells were treated with rapamycin and the expression of autophagic proteins and key components of Wnt signaling was evaluated. The ratio LC3BII/LC3BI was significantly increased (Fig. 4A and B) and P62 expression was significantly decreased in rMC-1 cells treated with rapamycin compared with those treated with vehicle (Fig. 4A and $\mathrm{C}$ ), suggesting that autophagy was induced in rMC-1 cells. Furthermore, protein expression of $n-p-\beta$-catenin was significantly increased in rapamycin-treated rMC-1 cells (Fig. 4A and D), whereas $\beta$-catenin expression was unchanged (Fig. 4A and E). Taken together, these results demonstrated that autophagy may activate Wnt signaling in rMC-1 cells.

Inhibition of autophagy downregulates Wnt signaling in rMC-1 cells. We treated rMC-1 cells with chloroquine, which is a commonly used autophagy inhibitor (25). Following chloroquine treatment, the ratio LC3BII/LC3BI was significantly decreased (Fig. 5A and B), whereas expression of P62 was significantly increased in rMC-1 cells (Fig. 5A and C). The expression of $n-p-\beta$-catenin was significantly decreased in rMC-1 cells treated with chloroquine compared with those treated with vehicle (Fig. 5A and D), whereas the protein expression of $\beta$-catenin was unchanged (Fig. 5A and E). Furthermore, the ratio LC3BII/LC3BI was significantly increased in high glucose-treated rMC-1 cells compared with control cells (Fig. 5F and G). In addition, the autophagy inhibitor 3-MA inhibited the upregulation of LC3BII/LC3BI in high glucose-treated rMC-1 cells (Fig. 5H and I), suggesting that autophagy may play a regulatory role through rMC-1 cells in diabetic retinas. 
A

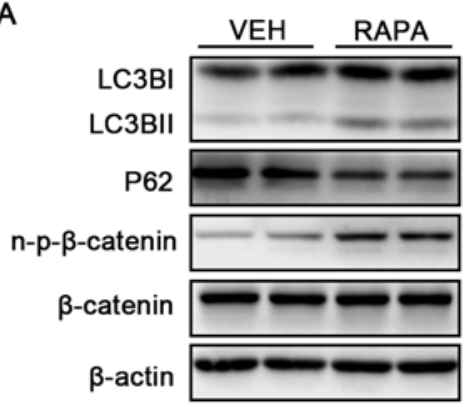

D

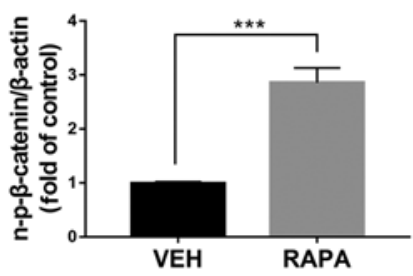

B

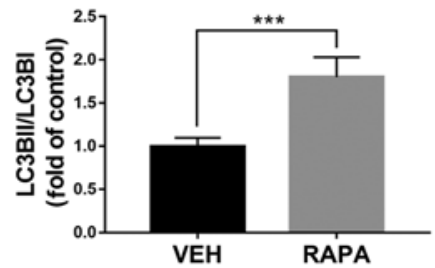

$\mathrm{E}$

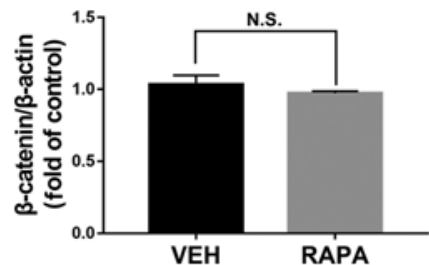

C

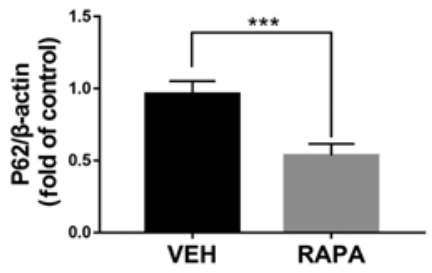

Figure 4. Induction of autophagy by RAPA upregulates Wnt signaling in rMC-1 cells. rMC-1 cells were treated with VEH or RAPA (4 $\mu \mathrm{M})$ for $24 \mathrm{~h}$. (A) Expression of LC3BI/II, P62,n-p- $\beta$-catenin and $\beta$-catenin was evaluated by western blotting. (B) Protein levels of LC3BI/II were quantified by densitometry and LC3BII/LC3BI was calculated and compared. (C-E) Expression of (C) P62, (D) n-p- $\beta$-catenin and (E) $\beta$-catenin was evaluated and quantified by densitometry and normalized to $\beta$-actin levels. All data are representative of three independent experiments. ${ }^{* * *} \mathrm{P}<0.001$. n-p- $\beta$-catenin, non-phosphorylated- $\beta$-catenin; N.S., non-significant; LC3B, microtubule-associated protein 1A/1B-light chain 3; VEH, vehicle; RAPA, rapamycin.

A

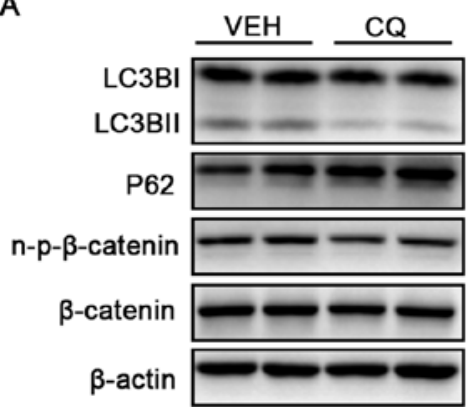

D

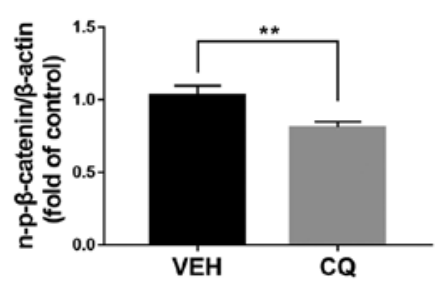

G

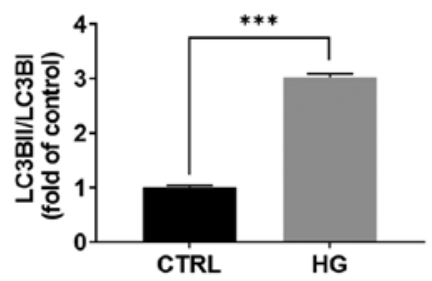

B

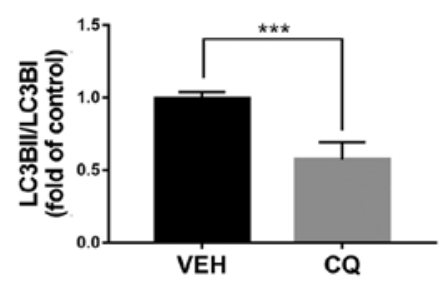

E.

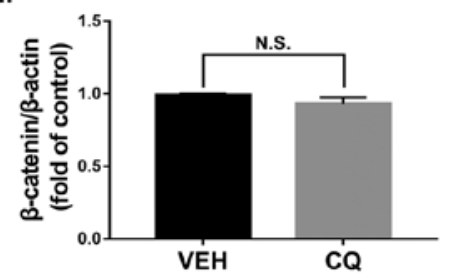

$\mathrm{H}$

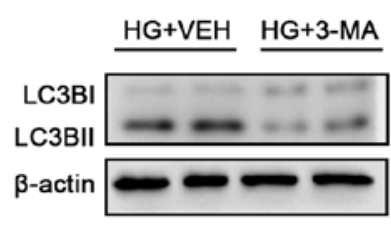

C

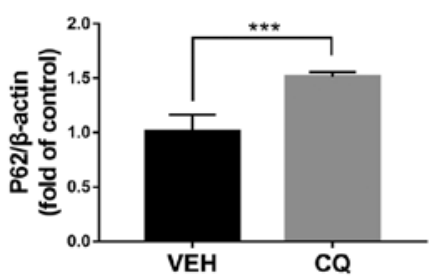

$\mathrm{F}$

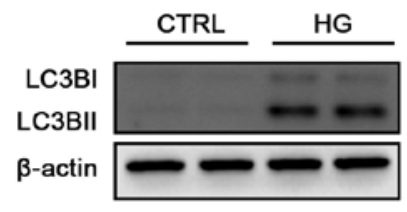

I

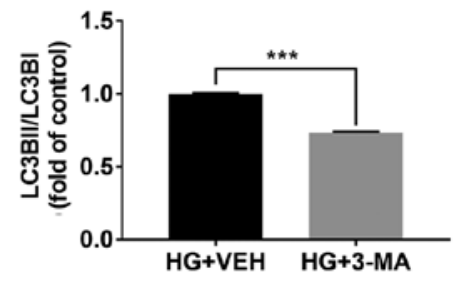

Figure 5. Inhibition of autophagy by CQ suppresses Wnt signaling in rMC-1 cells. rMC-1 cells were treated with VEH or CQ (1 $\mu \mathrm{M})$ for $24 \mathrm{~h}$. (A) Expression of LC3BI and LC3BI/II, P62, n-p- $\beta$-catenin and $\beta$-catenin was evaluated by western blotting. (B) Protein levels of LC3BI/II were quantified by densitometry and LC3BII/LC3BI was calculated and compared. Protein levels of (C) P62, (D) n-p- $\beta$-catenin and (E) $\beta$-catenin were quantified by densitometry and normalized to $\beta$-actin levels. (F amd G) Expression of LC3BI and LC3BII was evaluated by western blotting and quantified by densitometry. LC3BII/LC3BI was calculated and compared between CTRL rMC-1 cells and HG-treated cells. (H and I) Expression of LC3BI and LC3BII was evaluated by western blotting and quantified by densitometry. LC3BII/LC3BI was calculated and compared between rMC-1 cells treated with HG+VEH or HG+3-MA. All data are representative of three independent experiments. ${ }^{* *} \mathrm{P}<0.01$ and ${ }^{* * *} \mathrm{P}<0.001$. n-p- $\beta$-catenin, non-phosphorylated- $\beta$-catenin; N.S., non-significant; LC3B, microtubule-associated protein 1A/1B-light chain 3; VEH, vehicle; CQ, chloroquine; HG, high glucose; 3-MA, 3-methyladenin; CTRL, control. 


\section{Discussion}

Wnt signaling is involved in the physiological and pathological processes of a variety of diseases, including DR. The present study demonstrated that autophagy could positively regulate Wnt signaling in the retina of diabetic mice. Furthermore, pharmacological manipulation of autophagy could modulate Wnt signaling in the retina and retinal cells. To the best of our knowledge, the present study was the first to report the potential regulatory role of autophagy on Wnt signaling in the retina of diabetic mice. This study revealed a novel mechanism of Wnt signaling upregulation in DR by which autophagy may positively regulate Wnt signaling, indicating a possible new therapeutic target for DR via autophagy modulation.

Wnt signaling has been reported to contribute to the pathogenesis of DR (5); however, the mechanism of Wnt signaling upregulation in DR remains unknown. Previous studies demonstrated that autophagy might have a regulator role in Wnt signaling. For example, a previous study showed that nutrition starvation, a commonly used condition that induces autophagy in cells, downregulates Wnt signaling in HEK-293T cells; while the inhibition of autophagy with 3-MA attenuates the inhibitory effect of nutrition starvation on Wnt signaling (17). Furthermore, previous studies reported that autophagy is induced in the retina of diabetic human and mice $(13,14)$. In the present study, a regulatory role of autophagy on Wnt signaling in the retina of diabetic mice was demonstrated. Interestingly, unlike most of studies in cancer field which show a negative regulatory effect of autophagy on Wnt signaling $(17,26)$, our findings suggested that autophagy may positively regulate Wnt signaling in the retina of diabetic mice. A possible mechanism of autophagy negatively regulating Wnt signaling is that the autophagy protein P62 could bind to Dishevelled 2 (Dv12) and promote LC3-mediated autophagosome recruitment of Dvl2, thus accelerating Dvl degradation (17). However, only a few studies demonstrated that autophagy positively regulates Wnt signaling and described the possible underlying mechanism $(27,28)$. Further investigation is required to determine the regulatory mechanisms of autophagy on Wnt signaling in the retina, especially in the diabetic retina.

$\mathrm{Db} / \mathrm{db}$ mouse is a commonly used animal model of type 2 diabetes (29). Previous studies have shown that $\mathrm{db} / \mathrm{db}$ mice display pathological features of DR, such as loss of pericytes and retinal capillary degeneration $(30,31)$. In the present study, the ratio LC3BII/LC3BI and expression of Beclin-1 were increased, whereas P62 expression was decreased in the retinas of $\mathrm{db} / \mathrm{db}$ mice, indicating an activation of autophagy in diabetic retinas. These findings were consistent with other studies reporting that autophagy is induced in DR (12-14). In addition, the expression of a key component of Wnt signaling, n-p- $\beta$-catenin, and of the downstream target gene of Wnt signaling, VEGF, were increased in the present study, suggesting that Wnt signaling is activated in $\mathrm{db} / \mathrm{db}$ retinas. Activation of both autophagy and Wnt signaling in $\mathrm{db} / \mathrm{db}$ retinas indicates a possible relationship between them. Furthermore, Wnt signaling was inhibited in $\mathrm{db} / \mathrm{db}$ mice treated with the autophagy inhibitor 3-MA and activated after intraperitoneal injection of the autophagy activator rapamycin in normal C57BL/6J mice. These results suggested that autophagy could positively regulate Wnt signaling in diabetic retinas, and that modulating autophagy may control Wnt signaling in DR, which may serve as a potential therapeutic strategy for DR.

Müller cell, which is a major retinal glial cell type, covers the whole retina and interacts with many other types of cell in the retina, and serves therefore a crucial role in maintaining retinal hemostasis (32). The involvement of Müller cells in DR has been well studied. For example, Müller cells are activated in DR and produce inflammatory cytokines, resulting in cell apoptosis in diabetic retinas (23). Previous studies have reported that high glucose can promote mitochondrial dysfunction of Müller cells and induce cells apoptosis, contributing therefore to the pathogenesis of DR $(23,33)$. Furthermore, other studies have demonstrated that high glucose could induce autophagy in Müller cells $(14,34)$. Therefore, the present study used Müller cells to further investigate the relationship between autophagy and Wnt signaling. In this study, Wnt signaling was upregulated in rapamycin-treated Müller cells and inhibited in chloroquine-treated Müller cells. Interestingly, we found that chloroquine-induced changes in LC3II expression had a dose-dependent effect on Müller cells, and low dose of chloroquine could decrease LC3BII expression. Similar observation was found by Iwai-Kanai et al (35) in cardiac-derived myocytes. In addition, in the present study, high glucose could induce autophagy whereas 3-MA was capable of reversing the induction of autophagy in Müller cells. These findings demonstrated that manipulation of autophagy could modulate Wnt signaling in Müller cells, indicating autophagy may regulate Wnt signaling in Müller cells.

In the present study, the lack of immunostaining of autophagic markers in the retina was a limitation. Further investigation will explore the autophagic changes in specific retinal cells of diabetic retinas, especially in Müller cells.

In summary, the present study demonstrated that autophagy could positively regulate Wnt signaling in diabetic retinas. These findings revealed a potential role for autophagy in regulating Wnt signaling in DR, and modulation of autophagy may have therapeutic effects in DR.

\section{Acknowledgements}

We would like to thank Dr Vijay Sarthy at Northwestern University (Evanston, IL, USA) for providing the rMC-1 cell line.

\section{Funding}

This study was supported by the Natural Science Foundation of Fujian Province (grant no. 2019J01017), the National Science Foundation for Young Scientists of China (grant no. 31807795) and the National Key R\&D program of China (grant no. 2018YFA0107302).

\section{Availability of data and materials}

The datasets used and/or analyzed during the current study are available from the corresponding authors on reasonable request. 


\section{Authors' contributions}

SY, YZ, ZL and QC designed the experiments. SY, YZ, XW, XL, MW and RZ performed the experiments. SY, YZ and QC analyzed the data. ZL and QC supervised the experiments. SY, $\mathrm{ZL}$ and QC wrote the manuscript. QC revised the manuscript. SY and QC confirm the authenticity of all the raw data. All authors have read and approved the final manuscript.

\section{Ethics approval and consent to participate}

The animal protocols were approved by the Xiamen University Experimental Animal Ethics Committee (approval no. XMULA20190022).

\section{Patient consent for publication}

Not applicable.

\section{Competing interests}

The authors declare that they have no competing interests.

\section{References}

1. Wild S, Roglic G, Green A, Sicree R and King H: Global prevalence of diabetes: Estimates for the year 2000 and projections for 2030. Diabetes Care 27: 1047-1053, 2004.

2. Klein R, Knudtson MD, Lee KE, Gangnon R and Klein BE: The Wisconsin Epidemiologic Study of Diabetic Retinopathy: XXII the twenty-five-year progression of retinopathy in persons with type 1 diabetes. Ophthalmology 115: 1859-1868, 2008.

3. Ting DSW and Wong TY: Proliferative diabetic retinopathy: Laser or eye injection? Lancet 389: 2165-2166, 2017.

4. Clevers H: Wnt/beta-catenin signaling in development and disease. Cell 127: 469-480, 2006.

5. Chen Q and Ma JX: Canonical Wnt signaling in diabetic retinopathy. Vision Res 139: 47-58, 2017.

6. Clevers $\mathrm{H}$ and Nusse R: Wnt/ $\beta$-catenin signaling and disease. Cell 149: 1192-1205, 2012.

7. Chen Y, Hu Y, Zhou T, Zhou KK, Mott R, Wu M, Boulton M, Lyons TJ, Gao G and Ma JX: Activation of the Wnt pathway plays a pathogenic role in diabetic retinopathy in humans and animal models. Am J Pathol 175: 2676-2685, 2009.

8. Liu X, Zhang B, McBride JD, Zhou K, Lee K, Zhou Y, Liu Z and Ma JX: Antiangiogenic and antineuroinflammatory effects of kallistatin through interactions with the canonical Wnt pathway. Diabetes 62: 4228-4238, 2013

9. Lee K, Hu Y, Ding L, Chen Y, Takahashi Y, Mott R and Ma JX Therapeutic potential of a monoclonal antibody blocking the Wnt pathway in diabetic retinopathy. Diabetes 61: 2948-2957, 2012.

10. Eskelinen EL and Saftig P: Autophagy: A lysosomal degradation pathway with a central role in health and disease. Biochim Biophys Acta 1793: 664-673, 2009.

11. Boya P, Esteban-Martínez L, Serrano-Puebla A, Gómez-Sintes R and Villarejo-Zori B: Autophagy in the eye: Development, degeneration, and aging. Prog Retin Eye Res 55: 206-245, 2016.

12. Rosa MD, Distefano G, Gagliano C, Rusciano D and Malaguarnera L: Autophagy in Diabetic Retinopathy. Curr Neuropharmacol 14: 810-825, 2016.

13. Fu D, Yu JY, Yang S, Wu M, Hammad SM, Connell AR, Du M, Chen J and Lyons TJ: Survival or death: A dual role for autophagy in stress-induced pericyte loss in diabetic retinopathy. Diabetologia 59: 2251-2261, 2016.

14. Lopes de Faria JM, Duarte DA, Montemurro C, Papadimitriou A, Consonni SR and Lopes de Faria JB: Defective Autophagy in Diabetic Retinopathy. Invest Ophthalmol Vis Sci 57: 4356-4366, 2016.

15. Li P, Guo Y, Bledsoe G, Yang Z, Chao L and Chao J: Kallistatin induces breast cancer cell apoptosis and autophagy by modulating Wnt signaling and microRNA synthesis. Exp Cell Res 340: 305-314, 2016.
16. Peng Y, Cao J, Yao XY, Wang JX, Zhong MZ, Gan PP and Li JH: TUSC3 induces autophagy in human non-small cell lung cancer cells through Wnt/ $\beta$-catenin signaling. Oncotarget 8: 52960-52974, 2017.

17. Gao C, Cao W, Bao L, Zuo W, Xie G, Cai T, Fu W, Zhang J, Wu W, Zhang X, et al: Autophagy negatively regulates Wnt signalling by promoting Dishevelled degradation. Nat Cell Biol 12: 781-790, 2010.

18. Liu H, Lei H, Shi Y, Wang JJ, Chen N, Li ZH, Chen YF, Ye QF and Yang Y: Autophagy inhibitor 3-methyladenine alleviates overload-exercise-induced cardiac injury in rats. Acta Pharmacol Sin 38: 990-997, 2017.

19. James MH, Quinn RK, Ong LK, Levi EM, SmithDW, Dickson PW and Dayas CV: Rapamycin reduces motivated responding for cocaine and alters GluA1 expression in the ventral but not dorsal striatum. Eur J Pharmacol 784: 147-154, 2016.

20. Li X, Shan J, Chang W, Kim I, Bao J, Lee HJ, Zhang X, Samuel VT, Shulman GI, Liu D, et al: Chemical and genetic evidence for the involvement of Wnt antagonist Dickkopf 2 in regulation of glucose metabolism. Proc Natl Acad Sci USA 109: 11402-11407, 2012.

21. Chen Q, Qiu F, Zhou K, Matlock HG, Takahashi Y, Rajala RVS, Yang Y, Moran E and Ma JX: Pathogenic Role of microRNA-21 in Diabetic Retinopathy Through Downregulation of PPAR $\alpha$. Diabetes 66: 1671-1682, 2017.

22. Seglen PO and Gordon PB: 3-Methyladenine: Specific inhibitor of autophagic/lysosomal protein degradation in isolated rat hepatocytes. Proc Natl Acad Sci USA 79: 1889-1892, 1982.

23. Coughlin BA, Feenstra DJ and Mohr S: Müller cells and diabetic retinopathy. Vision Res 139: 93-100, 2017.

24. ZhouKK,BenyajatiS,Le Y,ChengR,Zhang W and MaJX:Interruption of Wnt signaling in Müller cells ameliorates ischemia-induced retinal neovascularization. PLoS One 9: e108454, 2014.

25. Mauthe M, Orhon I, Rocchi C, Zhou X, Luhr M, Hijlkema KJ, Coppes RP, Engedal N, Mari M and Reggiori F: Chloroquine inhibits autophagic flux by decreasing autophagosome-lysosome fusion. Autophagy 14: 1435-1455, 2018.

26. Cicchini M, Chakrabarti R, Kongara S, Price S, Nahar R, Lozy F, Zhong H, Vazquez A, Kang Y and Karantza V: Autophagy regulator BECN1 suppresses mammary tumorigenesis driven by WNT1 activation and following parity. Autophagy 10: 2036-2052, 2014.

27. Fan Q, Yang L, Zhang X, Ma Y, Li Y, Dong L, Zong Z, Hua X, $\mathrm{Su} \mathrm{D}, \mathrm{Li} \mathrm{H}$, et al: Autophagy promotes metastasis and glycolysis by upregulating MCT1 expression and $\mathrm{Wnt} / \beta$-catenin signaling pathway activation in hepatocellular carcinoma cells. J Exp Clin Cancer Res 37: 9, 2018.

28. Ríos JA, Godoy JA and Inestrosa NC: Wnt3a ligand facilitates autophagy in hippocampal neurons by modulating a novel GSK-3ß-AMPK axis. Cell Commun Signal 16: 15, 2018.

29. Burke SJ, Batdorf HM, Burk DH, Noland RC, Eder AE, Boulos MS, Karlstad MD and Collier JJ: db/db mice exhibit features of human type 2 diabetes that are not present in weight-matched C57BL/6J mice fed a Western diet. J Diabetes Res 2017: 8503754, 2017.

30. Bogdanov P, Corraliza L, Villena JA, Carvalho AR, Garcia-Arumí J, Ramos D, Ruberte J, Simó R and Hernández C: The $\mathrm{db} / \mathrm{db}$ mouse: A useful model for the study of diabetic retinal neurodegeneration. PLoS One 9: e97302, 2014.

31. Midena E, Segato T, Radin S, di Giorgio G, Meneghini F, Piermarocchi S and Belloni AS: Studies on the retina of the diabetic $\mathrm{db} / \mathrm{db}$ mouse. I. Endothelial cell-pericyte ratio. Ophthalmic Res 21: 106-111, 1989.

32. Bringmann A, Pannicke T, Grosche J, Francke M, Wiedemann P, Skatchkov SN, Osborne NN and Reichenbach A: Müller cells in the healthy and diseased retina. Prog Retin Eye Res 25: 397-424, 2006.

33. Tien T, Zhang J, Muto T, Kim D, Sarthy VP and Roy S: High Glucose Induces Mitochondrial Dysfunction in Retinal Müller Cells: Implications for Diabetic Retinopathy. Invest Ophthalmol Vis Sci 58: 2915-2921, 2017.

34. Ao H, Li H, Zhao X, Liu B and Lu L: TXNIP positively regulates the autophagy and apoptosis in the rat müller cell of diabetic retinopathy. Life Sci 267: 118988, 2021.

35. Iwai-Kanai E, Yuan H, Huang C, Sayen MR, Perry-Garza CN, Kim L and Gottlieb RA: A method to measure cardiac autophagic flux in vivo. Autophagy 4: 322-329, 2008.

This work is licensed under a Creative Commons Attribution-NonCommercial-NoDerivatives 4.0 International (CC BY-NC-ND 4.0) License. 